\title{
Analysis of vibration energy harvester with magnetic spring
}

\author{
Marcin Kulik $^{1, *}$, Rafał Gabor ${ }^{1}$, and Mariusz Jagieła ${ }^{1}$ \\ ${ }^{1}$ Opole University of Technology, Faculty of Electrical Engineering, Automatic Control and Informatics, Opole, Poland
}

\begin{abstract}
In paper an electromagnetic energy harvester with two fixed permanent magnets and one moving suspended vertically by the magnetic interaction between them. The external force imposed on the system causes displacement of the magnet whose magnetic field induces emf in the coil wound around it. In order to obtain the resonance frequency around $25 \mathrm{~Hz}$, the numerical optimisation was carried out. The measured frequency characteristics of the manufactured system compare well with results of computer simulation.
\end{abstract}

\section{Introduction}

In recent years, the problems involving obtaining electrical energy from the environment with its smart management have become research areas [1-3]. The process itself involves harvesting this energy from sources like wind, sunlight, heat or vibrations, and storing it in capacities or batteries [2]. This work concentrates on the mechanical vibrations.

\section{Electromagnetic vibration energy harvester}

Due to simplicity and relatively high induced voltage, the electromagnetic harvester shown in Figure 1 is proposed. It is a coreless system with the magnetic circuit composed of permanent magnets. The magnetic spring formed by the magnetically suspended central magnet ring brings a nonlinearity due to dependence of the magnetic force $f_{\text {mag }}$ on displacement $x$ which extends the operation frequency bandwidth of the considered system.

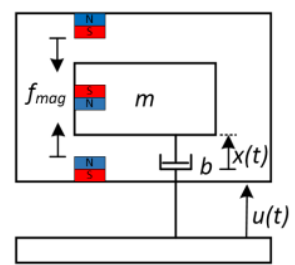

b)

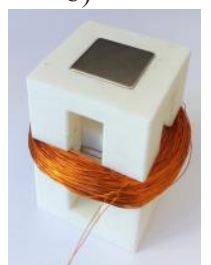

Fig.1. Vibration energy harvester, a) kinematic diagram, with $b$-damping coefficient, $m$ - mass of moving element, $u(t)-$ displacement of the source of vibrations, b) manufactured prototype with coil wound around the moving magnet.

Under the influence of external vibrations the suspended magnet moves relative to the coil, and this induces emf in the coil. After linearization of the magnetic force, the equation of motion of the system in Fig. 1 reads

$$
m \ddot{x}(t)=m \ddot{u}(t)-\bar{k} x(t)-b \dot{x}(t)
$$

where: $\dot{x}$ and $\ddot{x}$ are velocity and acceleration of the mass $m$, whilst $\ddot{u}$ is the acceleration of force applied to the casing, and $k$ is a magnetic stiffness linearized around $x=0$. A more accurate analysis, should however take the $k$ as a function of displacement into account, which would results in the occurrence of non-linear resonance $[1,3]$.

\section{Designing of the system}

The casing of the converter is manufactured of a plastic. The two fixed magnets are placed on the peripheries of the casing. In order to allow for the magnetic suspension of the moving magnet the poles are oriented as shown in Fig. 2. The moving magnet is also stabilised mechanically by the guides imprinted in the walls of casing. a)

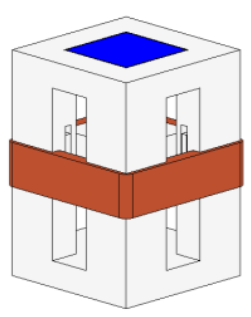

b)

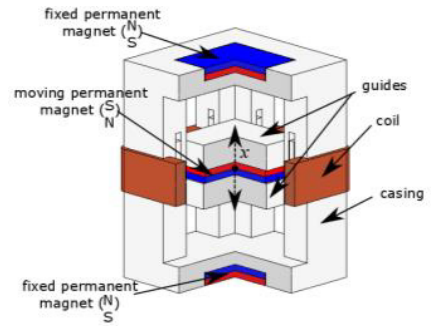

Fig.2. Tubular electromagnetic energy harvester model: a) CAD drawing and b) view of cross-section 3/4.

In the first stage, the converter dimensions were optimized in order to obtain the main resonant frequency equal to $25 \mathrm{~Hz}$. The value of the stiffness coefficient necessary to meet the assumption can be determined from usual formula 


$$
\bar{k}=\left(2 \cdot \pi \cdot f_{\text {res }}\right)^{2} \cdot m
$$

where $f_{\text {res }}$ is the resonant frequency, $m=0.01 \mathrm{~kg}$, and

$$
\bar{k}_{\text {mag }}=\frac{d f_{\text {mag }}}{d x \quad x=0} \approx \frac{f_{\text {mag }}(\Delta x)-f_{\text {mag }}(-\Delta x)}{2 \Delta x}
$$

where $f_{\text {mag }}$ is calculated from the Maxwell stress tensor method through the finite element analysis of the magnetic field distribution [4], $\Delta x$ is the assumed virtual displacement the moving magnet. Optimization was carried out using the genetic algorithm from the Direct Search Matlab Toolbox [5]. The goal function was to obtain the value of $\bar{k}_{\text {mag }}$ that ensures minimum of square norm of the residuum of equation (2). The design variable was the distance between the fixed and moving magnets, which for the remaining dimensions of the system being constant, has the greatest impact on magnetic force. The variation of the variable was between $35 \mathrm{~mm}$ and $120 \mathrm{~mm}$. After calculations, the optimal distance between permanent magnets was obtained, which was equal to $45 \mathrm{~mm}$.

\section{Computer simulation}

The simulation of the tested system was carried with the purpose of determining its frequency-response characteristic. The latter was determined by solution of equation (1) within a time-interval of $400 \mathrm{~s}$ as the magnitude of the excitation system $\ddot{u}$ was kept constant while the frequency was swept within between 5 and 60 $\mathrm{Hz}$. The variations of the flux linkage and force $f_{\text {mag }}$ vs. displacement $x$ were taken from the finite element analysis. The simulated frequency characteristics of the induced emf for different magnitudes of the excitation force $f_{\text {exc }}=m \ddot{u}$ are shown in Fig.3. It can be seen from figure that as the the magnitude of the external force increases, the resonant frequency shifts towards higher values, which is clearly the effect of rise of the magnetic stiffness of the system for large displacements. For the studied cases, the frequency range where the induced voltage is relatively large is between 18.2 and $25.4 \mathrm{~Hz}$. The maximum magnitude of the induced emf was equal to $2.2 \mathrm{~V}$.

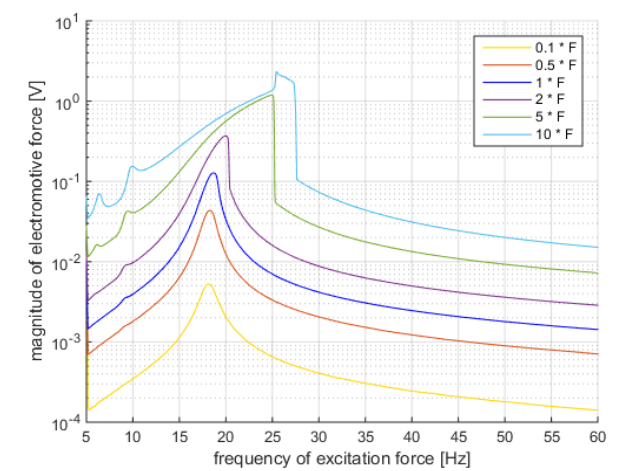

Fig.3. Frequency characteristics for electromotive force at noload condition for different magnitudes of excitation force, where $\mathrm{F}=0.0424 \mathrm{~N}$.

\section{Physical validation}

The measurements were carried out on a laboratory teststand shown in Fig. 4a. The voltage values were read at frequencies between $15 \mathrm{~Hz}$ and $30 \mathrm{~Hz}$ for the magnitude of the excitation equal to $0.0424 \mathrm{~N}$. Fig. $4 \mathrm{~b}$ compares the measured waveform with that obtained from simulation.

a)

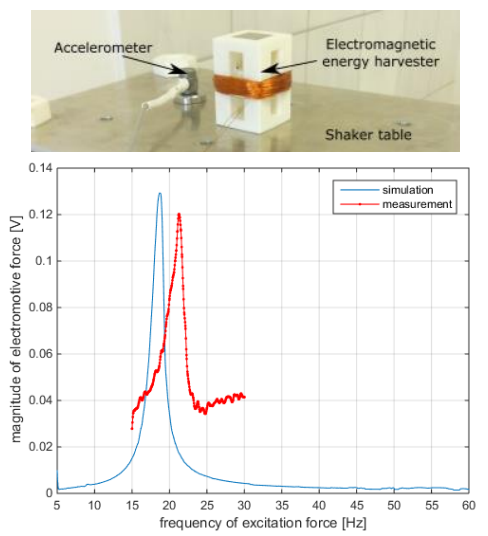

Fig.4. a) Laboratory test-stand, b) comparison of measured and simulated frequency characteristics of induced emf for magnitude of excitation force equal to $0.0424 \mathrm{~N}$.

The comparison clearly shows the impact of the friction force, which is very difficult for identification due to complex type and event a discontinuity.

\section{Conclusion}

In this work an electromagnetic vibration energy harvester was designed and analysed. The magnetic spring contained in the system is capable to bring only the positive magnetic stiffness effect, and consequently the system should operate only in the low frequency range.

This work was carried out under project 2016/23/N/ST7/03808 of the National Science Centre, Poland

\section{References}

1. T. Kaźmierski, S. Beeby, Energy Harvesting Systems, Principles, Modeling, Applications, Springer, 2011

2. K. Kecik, A. Mitura, S. Lenci, J. Warminski, Int J Non Linear Mech, 1-7(2017)

3. M. Jagieła, M. Kulik, Prz. Elektrotech. 1(93), 311315 (2017)

4. K. Adamiak, J. Mizia, G. Dawson, A. Eastham, IEEE Transactions on Magnetics 23, 3005-3007 (1987)

5. D.E. Goldberg, Genetic Algorithms in Search, Optimization \& Machine Learning (AddisonWesley, 1989) 